Available online at GSC Online Press Directory

GSC Advanced Research and Reviews e-ISSN: 2582-4597, CODEN (USA): GARRC2

Journal homepage: https://www.gsconlinepress.com/journals/gscarr

(RESEARCH ARTICLE)

\title{
Testing the efficacy of Mueller Hinton agar over Nutrient agar for optimal antibiotic sensitivity testing response by selected clinical bacterial pathogens
}

\author{
Otajevwo Dafinone Festus * and Osawaru Osama Emmanuella \\ Department of Microbiology, Western Delta University, Oghara, Nigeria.
}

Publication history: Received on 14 May 2020; revised on 22 June 2020; accepted on 13 November 2020

Article DOI: https://doi.org/10.30574/gscarr.2020.5.2.0037

\begin{abstract}
The efficacy of Mueller Hinton agar over Nutrient agar in terms of antibiotic sensitivity testing for optimal antibiotic response by selected clinical bacterial pathogens was carried out in this study. Clinical bacterial pathogens used for the study were Pseudomonas aeruginosa, Enterococcus spp, Escherichia coli and Klebsiella pneumoniae. Standard and locally manufactured antibiotic discs used were by Abtek Biologicals Ltd, Liverpool and Maxicare Medical Laboratory, Nigeria respectively. Antibiotic sensitivity testing (AST) was by agar diffusion method. Pure cultures of each isolate were subcultured on sterile Mueller Hinton agar (MHA) and Nutrient agar (NA) media after which the standard and locally manufactured discs were aseptically impregnated on the media. All inoculated plates were incubated at $37^{\circ} \mathrm{C}$ for $24 \mathrm{hrs}$ aerobically after appropriate labeling. Zones of inhibition were measured by standard methods and recorded. On Nutrient agar, standard and locally produced ciprofloxacin, ofloxacin, gentamycin and amoxicillin/clavulanic acid discs did not produce zones of inhibition significantly different from each other at both $95 \%$ and $99 \%$ confidence intervals (P $>0.05$ and $\mathrm{P}>0.01$ ). On Mueller Hinton agar, standard and locally manufactured ciprofloxacin, ofloxacin, gentamycin and amoxicillin/clavulanic acid discs produced zones of inhibition that were significantly different from each other at 95\% confidence interval $(\mathrm{P}<0.05)$. Standard and local ciprofloxacin, ofloxacin, gentamycin and amoxicillin/clavulanic acid discs produced zones of inhibition on MHA and NA which were not significantly different $(\mathrm{P}>0.05$ and $\mathrm{P}>0.01)$. Standard discs used recorded better zones of inhibition on MHA compared to the local discs. Standard and local discs zones of inhibition on MHA was however not significantly different from those recorded on NA (P > 0.05). Standard discs therefore, did not produce better zones of inhibition over local discs on MHA and on NA. On the whole, the use of MHA for antibiotic sensitivity testing did not record greater (better) zones of inhibition than those recorded on NA except for standard ciprofloxacin, ofloxacin and gentamycin discs over the corresponding local discs on MHA only. Findings did not convincingly establish better performance of standard discs over local discs whether used on MHA or NA. Further studies in this direction is recommended.
\end{abstract}

Keywords: AST; Efficacy; MHA; NA; Optimal Response; Bacterial Pathogens.

\section{Introduction}

Antibiotic resistance has become a serious public health problem all over the world. Nearly two million people in the USA acquire nosocomial infections every year, resulting in 90,000 deaths [1]. More than $70 \%$ of the bacteria that cause these infections are resistant to at least one of the antibiotics commonly used in treatment [2]. This makes the selection of an appropriate agent an increasingly more challenging task that has made clinicians more dependent on data from in-vitro AST [3].

\footnotetext{
${ }^{*}$ Corresponding author: Otajevwo Dafinone Festus

Department of Microbiology, Western Delta University, Oghara, Nigeria. 
The selection of an appropriate antimicrobial agent is a challenging task for clinicians [1]. The Kirby-Bauer disk diffusion method is one of the most widely practiced antimicrobial susceptibility tests (AST). It is affected by many factors among which are the media used. Mueller-Hinton agar (MHA) is the standard medium recommended in guidelines. However, these guidelines are not strictly adhered to in some developing countries.

In the industrial world, the Kirby-Bauer disk diffusion method is a standard procedure for the susceptibility testing of bacterial isolates. When the test is performed following a standard procedure, it gives reliable results and can predict clinical efficacy of the antibiotics tested $[4,5]$. It has been recognized for years that the general adoption of antimicrobial susceptibility test (AST) method, so standardized as to minimize the influence of variables, would be a great advance. The validity of AST depends on rigid standardization of every feature of the test particularly the composition of the medium used [6]. The standard medium for the Kirby-Bauer method of susceptibility testing is Mueller-Hinton agar [5]. Because of the number of difficulties and financial issues, MHA is not a feasible option in many developing countries and instead, NA is used for AST [7].

Mueller-Hinton agar is a loose agar that allows for better diffusion of the antibiotics than most other media and a better diffusion leads to a truer zone of inhibition [8]. This criterion was missing in NA manifested by smaller IZs with TOB, $\mathrm{CN}$, and P when testing P. aeruginosa ATCC 27853, AZM and DA when testing S. aureus ATCC 25923 as well as AMP, KZ, TOB, CN when testing E. coli ATCC 25922. Both the para-aminobenzoic acid (PABA) and thymine/thymidine content in MHA are reduced to a minimum, thus markedly reducing the inactivation of sulfonamides and trimethoprim when the medium is used for testing the susceptibility of bacterial isolates to these antimicrobials [8]. Thirteen errors with smaller inhibition zone over 30 consecutive days were detected on testing SXT for S. aureus ATCC 25923 using NA.

The inability of AST to determine a susceptible result for an organism that is susceptible to the antimicrobial agent being tested is considered a major error or false resistant [9]. Conversely, the inability to detect resistance is assessed as "very major error" or false sensitive [9]. Concerning CLSI guidelines, there is a minimum level of acceptable interpretative errors in susceptibility testing which are quite restrictive. Very major errors should not exceed $1.5 \%$, while major errors should not exceed 3.0\%, and overall categorical agreement should equal or exceed $90 \%$ for each organism antibiotic challenge $[10,9,11]$.

According to Nassar et al. [1], all antimicrobial agent-reference organism combinations using NA were unacceptable compared to MHA. All antibiotic-organism combinations showed more than three misreading over 30 successive days. Hence, none of the results were accepted according to CLSI guidelines. The discrepancies between the susceptibility results obtained by NA and the standard MHA were obvious when testing clinical isolates with total errors of $27.76 \%$, $22.4 \%$, and 3.6\% with P. aeruginosa, S. aureus, and Enterobacteriaceae respectively. Also, very major errors and major errors were beyond the acceptable level of CLSI guidelines (8.98\% and 7.6\% very major errors and $4.08 \%$ and $6 \%$ major errors for P. aeruginosa and S. aureus isolates respectively).

Very major error may lead to the initiation of inadequate antimicrobial therapy and may have fatal consequences especially in severely ill patients where these antibiotics are common first-line substances. On the other hand, major errors deprive the patients of treatment with an effective antibiotic and lead to the use of second-choice drugs, usually more recent and expensive, and thus contribute to economic losses and the selection of resistant strains [9].

Although none of the AST results for E. coli ATCC 25922 was acceptable when using NA, a remarkable unexplained agreement in AST results of Enterobacteriaceae on both NA and MHA (96.4\%) was found with no very major error and $0.4 \%$ major error [1]. Even if the AST results showed full agreement as in the case of Enterobacteriaceae, the issue of lacking data on specific breakpoints concerning the use of NA remains.

Owing to the fact that NA is a general purpose medium rather than standard susceptibility testing medium, there is hardly any data comparing it with MHA in susceptibility testing. Donkor et al. [12] compared NA with MHA in antimicrobial susceptibility testing of Salmonella typhi and S. aureus isolates. They reported that the overall discrepancy in susceptibility results between NA and MHA was 8.9\%, and thus discouraged use of NA in AST due to the considerable error margin this medium may introduce into susceptibility results [12]. This study seeks to provide an update on the overall performance of MHA over NA with regard to antibiotic sensitivity testing (AST). The aim therefore, of this work is to test the efficacy of Mueller Hinton agar over Nutrient agar for optimal antibiotic response by selected clinical bacterial pathogens with the below objectives:

To determine local and standard antibiotic sensitivity testing patterns of selected pathogens on sterile Nutrient agar and Mueller Hinton agar. 
To statistically compare means of zones of inhibition of standard and local discs on Nutrient agar and Mueller Hinton agar.

To statistically compare means of standard and local discs zones of inhibition on Mueller Hinton agar (MHA) only.

To statistically compare means of standard and local discs zones of inhibition on Nutrient agar (NA) only.

To statistically compare means of standard and local disc zones of inhibition on MHA and NA.

\section{Material and methods}

\subsection{Sampling/Processing of Samples}

Four pure (axenic) cultures or clinical isolates of Pseudomonas aeruginosa, Enterococcus spp, Escherichia coli and Klebsiella pneumoniae were obtained courtesy of the Pharmaceutical Microbiology Department of the Faculty of Pharmacy, University of Benin, Nigeria. Isolates were stored on appropriately labeled sterile Nutrient agar slants and taken to the Microbiology section of Chrida Medical laboratory based in Benin City, Nigeria.

Inoculated slant cultures were incubated aerobically at $37^{\circ} \mathrm{C}$ for $24 \mathrm{hrs}$. The purity of the isolates was determined by Gram staining, morphological, biochemical and sugar fermentation characterization tests by standard methods [13,14]. Confirmed cultures were kept at $4^{\circ} \mathrm{C}$ in a refrigerator for further use after appropriate labeling. The four selected bacterial pathogens were then subjected to antibiotic sensitivity testing (AST) with local and standard antibiotic discs.

\subsection{Local Antibiotic Discs}

The local antibiotic discs used were Gram negative discs obtained from Maxicare Medical laboratory, Nigeria and contained septrin $(30 \mu \mathrm{g})$, chloramphenicol $(30 \mu \mathrm{g})$, sparfloxacin $(10 \mu \mathrm{g})$, ciprofloxacin $(30 \mu \mathrm{g})$, amoxicillin $(30 \mu \mathrm{g})$, amoxicillin/clavulanic acid or a $\mu$ gmentin $(10 \mu \mathrm{g})$, gentamycin $(30 \mu \mathrm{g})$, perfloxacin $(30 \mu \mathrm{g})$, ofloxacin or tarivid $(10 \mu \mathrm{g})$ and streptomycin $(30 \mu \mathrm{g})$.

\subsection{Standard Antibiotic Discs}

Gram negative discs manufactured by Abtek Biologicals Ltd, Liverpool, were used and the discs contained ceftazidime $(30 \mu \mathrm{g})$, cefuroxime $(30 \mu \mathrm{g})$, gentamycin $(10 \mu \mathrm{g})$, cefixime $(5 \mu \mathrm{g})$, ofloxacin $(5 \mu \mathrm{g})$, amoxicillin/clavulanic acid $(30 \mu \mathrm{g})$, nitrofurantoin $(300 \mu \mathrm{g})$ and ciprofloxacin $(5 \mu \mathrm{g})$.

\subsection{Antibiotic Sensitivity Testing}

Antibiotic sensitivity testing was carried out by agar diffusion according to the method prescribed by Bauer et al. [15]. The slant culture of each isolate was subcultured aseptically on sterile Nutrient agar (LabM, UK) plates in duplicate using a flamed and cooled inoculating wire loop. The same isolate was also aseptically subcultured on sterile Mueller Hinton agar (Titan Biotech Ltd, India) plates in duplicate. The plates were subcultured by first placing the culture loop in the centre of the plate and spreading from the centre all over the plates applying the caution of not touching the edges of the plates.

The seeded plates were allowed to stand for about 2 minutes to allow the agar surface to dry. A pair of forceps was flamed and cooled and used to pick the local multidiscs and then impregnated on the sterile Nutrient agar (NA) plates as well as the Mueller Hinton agar (MHA) plates. All plates were appropriately labeled. The same pair of forceps were flamed and cooled and used to pick the standard multidiscs which were impregnated on separate NA and MHA plates after proper labeling.

The discs were placed at least $22.0 \mathrm{~mm}$ from each other and $14.0 \mathrm{~mm}$ from the edge of the plates [16]. Antibiotic discs were selected on the basis of their clinical importance and efficacy on the selected pathogens. The seeded plates were allowed to stand for $10 \mathrm{mins}$ before incubation. At the end of incubation, the diameters of the zones of inhibition from one edge to the opposite edge were measured to the nearest millimeter using a transparent ruler [17]. 


\subsection{Comparing Mean Zones of Inhibition}

The mean zones of inhibition of all four pathogens to each antibiotic on NA and MHA were separately computed for. For further analysis, the antibiotics (to which all pathogens were sensitive) common to both local and standard disc panels on NA and MHA were selected and analysed statistically.

\subsection{Statistical Analyses}

Statistical analyses done on generated data was mean \pm standard error zones of inhibition. Paired T- test analyses were also carried at both $95 \%$ and $99 \%$ confidence intervals.

\subsection{Hypotheses Testing}

Based on the data generated, the following hypotheses were tested:

Zones of inhibition on MHA and NA by standard discs ARE NOT significantly different from each other. (NULL HYOTHESIS).

Zones of inhibition on MHA and NA by standard discs ARE significantly different from each other. (ALTERNATE HYOTHESIS).

Zones of inhibition produced on MHA and NA by local discs ARE NOT significantly different from each other (NULL HYOTHESIS).

Zones of inhibition produced on MHA and NA by local discs ARE significantly different from each other (ALTERNATE HYOTHESIS).

Zones of inhibition produced on MHA by standard and local discs ARE NOT significantly different from each other (NULL HYOTHESIS).

Zones of inhibition produced on MHA by standard and local discs ARE significantly different from each other (ALTERNATE HYOTHESIS).

Zones of inhibition produced on NA by standard and local discs ARE NOT significantly different from each other (NULL HYOTHESIS).

Zones of inhibition produced on NA by standard and local discs ARE significantly different from each other (ALTERNATE HYOTHESIS).

\section{Results}

The antibiotic sensitivity reports of Pseudomonas aeruginosa, Enterococcus spp, Escherichia coli and Klebsiella pneumoniae to the local discs (Maxicare Medical laboratory. Nigeria) and to the standard discs (Abtek Biologicals, Liverpool) are presented in Table 1. The antibiograms of the local and standard discs on sterile Nutrient agar as they relate to the above mentioned clinical pathogens are also presented in Table 1.

The antibiotic sensitivity reports of Pseudomonas aeruginosa, Enterococcus spp, Escherichia coli and Klebsiella pneumoniae to the local discs (Maxicare Medical laboratory. Nigeria) and to the standard discs (Abtek Biologicals, Liverpool) are also presented in Table 2. The antibiograms of the local and standard discs on sterile Mueller Hinton agar as they relate to the above mentioned clinical pathogens are also presented in Table 2. 
Table 1 Local and Standard Antibiotic Sensitivity Testing Patterns of Selected Pathogens on Sterile Nutrient agar

\begin{tabular}{|c|c|c|c|c|c|c|c|c|c|c|c|c|c|c|c|c|c|c|c|}
\hline \multicolumn{20}{|c|}{ Antibiotic Sensitivity Testing Patterns on nutrient agar } \\
\hline \multirow[b]{2}{*}{ Isolates } & \multirow{2}{*}{$\begin{array}{c}\text { Zones of } \\
\text { inhibition } \\
\text { Replicate }\end{array}$} & \multicolumn{10}{|c|}{ Local discs } & \multicolumn{8}{|c|}{ Standard discs } \\
\hline & & $\begin{array}{l}\text { SXT } \\
\mathrm{mm}\end{array}$ & $\begin{array}{l}\text { CHL } \\
\mathrm{Mm}\end{array}$ & $\begin{array}{c}\mathrm{SP} \\
\mathrm{mm}\end{array}$ & $\begin{array}{l}\mathrm{CPX} \\
\mathrm{mm}\end{array}$ & $\begin{array}{l}\mathrm{AM} \\
\mathrm{mm}\end{array}$ & $\begin{array}{l}\mathrm{AU} \\
\mathrm{mm}\end{array}$ & $\begin{array}{l}\mathrm{GN} \\
\mathrm{mm}\end{array}$ & $\begin{array}{l}\text { PEF } \\
\mathrm{mm}\end{array}$ & $\begin{array}{l}\text { OFX } \\
\mathrm{mm}\end{array}$ & $\begin{array}{c}\mathrm{ST} \\
\mathrm{Mm}\end{array}$ & $\begin{array}{l}\text { CAZ } \\
\mathrm{mm}\end{array}$ & $\begin{array}{l}\text { CRX } \\
\mathrm{mm}\end{array}$ & $\begin{array}{l}\mathrm{GN} \\
\mathrm{mm}\end{array}$ & $\begin{array}{l}\text { CXM } \\
\mathrm{mm}\end{array}$ & $\begin{array}{l}\text { OFL } \\
\mathrm{mm}\end{array}$ & $\begin{array}{l}\mathrm{A} \mu \mathrm{G} \\
\mathrm{mm}\end{array}$ & $\begin{array}{l}\text { NIT } \\
\mathrm{mm}\end{array}$ & $\begin{array}{l}\mathrm{CPR} \\
\mathrm{mm}\end{array}$ \\
\hline \multirow[b]{2}{*}{ PA } & $\mathrm{ZI}_{1}$ & 0.0 & 2.0 & 17.0 & 17.0 & 10.0 & 0.0 & 0.0 & 6.0 & 18.0 & 0.0 & 0.0 & 2.0 & 11.0 & 0.0 & 19.0 & 0.0 & 3.0 & 30.0 \\
\hline & $\mathrm{ZI}_{2}$ & 5.0 & 1.0 & 16.0 & 13.0 & 4.0 & 0.0 & 0.0 & 11.0 & 16.0 & 0.0 & 0.0 & 5.0 & 14.0 & 0.0 & 22.0 & 3.0 & 0.0 & 28.0 \\
\hline \multirow[b]{2}{*}{ ENT } & $\mathrm{ZI}_{1}$ & 3.0 & 13.0 & 11.0 & 12.0 & 3.0 & 0.0 & 6.0 & 10.0 & 11.0 & 0.0 & 0.0 & 3.0 & 10.0 & 0.0 & 19.0 & 5.0 & 3.0 & 15.0 \\
\hline & $\mathrm{ZI}_{2}$ & 7.0 & 9.0 & 13.0 & 14.0 & 0.0 & 3.0 & 8.0 & 7.0 & 13.0 & 0.0 & 0.0 & 0.0 & 13.0 & 0.0 & 17.0 & 5.0 & 4.0 & 21.0 \\
\hline \multirow[b]{2}{*}{ EC } & $\mathrm{ZI}_{1}$ & 0.0 & 0.0 & 14.0 & 10.0 & 0.0 & 0.0 & 0.0 & 6.0 & 14.0 & 0.0 & 0.0 & 2.0 & 10.0 & 0.0 & 16.0 & 0.0 & 0.0 & 10.0 \\
\hline & $\mathrm{ZI}_{2}$ & 0.0 & 0.0 & 0.0 & 0.0 & 3.0 & 0.0 & 0.0 & 11.0 & 15.0 & 0.0 & 0.0 & 2.0 & 0.0 & 0.0 & 15.0 & 0.0 & 0.0 & 21.0 \\
\hline \multirow{4}{*}{ KP } & $\mathrm{ZI}_{1}$ & 0.0 & 2.0 & 0.0 & 3.0 & 0.0 & 0.0 & 1.0 & 3.0 & 5.0 & 0.0 & 0.0 & 1.0 & 4.0 & 0.0 & 17.0 & 0.0 & 20.0 & 25.0 \\
\hline & $\mathrm{ZI}_{2}$ & 0.0 & 2.0 & 0.0 & 5.0 & 0.0 & 0.0 & 2.0 & 1.0 & 6.0 & 0.0 & 0.0 & 0.0 & 0.0 & 0.0 & 17.0 & 0.0 & 22.0 & 21.0 \\
\hline & $\begin{array}{c}\text { Mean } \\
\text { ZI }\end{array}$ & 1.88 & 3.63 & 8.88 & 9.25 & 2.50 & 0.38 & 2.13 & 6.88 & 12.25 & 0.0 & 0.0 & 1.88 & 7.75 & 0.0 & 17.75 & 1.63 & 6.50 & 21.38 \\
\hline & \multicolumn{11}{|c|}{$5.31 \mathrm{~mm}$} & \multicolumn{8}{|c|}{$8.13 \mathrm{~mm}$} \\
\hline
\end{tabular}

SXT=septrin, GN= gentamycin, $\mathrm{CHL}=$ chloramphenicol, $\mathrm{CPX}=$ ciprofloxacin, $\mathrm{AM}=$ amoxicillin, $\mathrm{ST}=$ streptomycin $\mathrm{CAZ}=$ ceftazidime, $\mathrm{CRX}=$ cefuroxime, $\mathrm{CXM}=$ cefixime, $\mathrm{OFL}=\mathrm{ofloxacin}$

$\mathrm{A} \mu \mathrm{G}=$ augmentin(amoxicillin/clavulanic acid, NIT=nitrofurantoin, $\mathrm{CPR}=$ ciprofloxacin,PEF=Pefloxacin, ZI = Zone of inhibition, $\mathrm{PA}$ (Pseudomonas aeruginsa), ENT (Enterococcus spp), EC (Escherichia coli), KP (Klebsiella pneumoniae). 
Table 2 Local and Standard Antibiotic Sensitivity Testing Patterns of Selected Pathogens on Sterile Mueller Hinton agar

\begin{tabular}{|c|c|c|c|c|c|c|c|c|c|c|c|c|c|c|c|c|c|c|c|}
\hline \multicolumn{20}{|c|}{ Antibiotic Sensitivity Testing Patterns on MUELLER HINTON AGAR } \\
\hline ISOLATES & $\begin{array}{l}\text { ZONES OF } \\
\text { INHIBITION } \\
\text { REPLICATE }\end{array}$ & $\begin{array}{l}\text { SXT } \\
\mathrm{Mm}\end{array}$ & $\begin{array}{l}\text { CHL } \\
\mathrm{mm}\end{array}$ & $\begin{array}{l}\mathrm{SP} \\
\mathrm{Mm}\end{array}$ & $\begin{array}{l}\mathrm{CPX} \\
\mathrm{mm}\end{array}$ & $\begin{array}{l}\mathrm{AM} \\
\mathrm{Mm}\end{array}$ & $\begin{array}{l}\mathrm{AU} \\
\mathrm{mm}\end{array}$ & $\begin{array}{l}\mathrm{GN} \\
\mathrm{Mm}\end{array}$ & $\begin{array}{l}\text { PEF } \\
\mathrm{mm}\end{array}$ & $\begin{array}{l}\text { OFX } \\
\mathrm{mm}\end{array}$ & $\begin{array}{l}\mathrm{ST} \\
\mathrm{mm}\end{array}$ & $\begin{array}{l}\text { CAZ } \\
\mathrm{mm}\end{array}$ & $\begin{array}{l}\text { CRX } \\
\mathrm{mm}\end{array}$ & $\begin{array}{l}\mathrm{GN} \\
\mathrm{mm}\end{array}$ & $\begin{array}{l}\text { CXM } \\
\mathrm{mm}\end{array}$ & $\begin{array}{l}\mathrm{OFL} \\
\mathrm{mm}\end{array}$ & $\begin{array}{l}\mathrm{A} \mu \mathrm{G} \\
\mathrm{mm}\end{array}$ & $\begin{array}{l}\text { NIT } \\
\mathrm{mm}\end{array}$ & $\begin{array}{l}\mathrm{CPR} \\
\mathrm{mm}\end{array}$ \\
\hline \multirow[b]{2}{*}{ PA } & $\mathrm{ZI}_{1}$ & 0.0 & 2.0 & 0.0 & 17.0 & 10.0 & 0.0 & 5.0 & 13.0 & 18.0 & 0.0 & 0.0 & 1.0 & 17.0 & 0.0 & 20.0 & 5.0 & 7.0 & 30.0 \\
\hline & $\mathrm{ZI}_{2}$ & 4.0 & 6.0 & 3.0 & 18.0 & 2.0 & 0.0 & 0.0 & 15.0 & 18.0 & 0.0 & 2.0 & 1.0 & 19.0 & 0.0 & 20.0 & 0.0 & 3.0 & 31.0 \\
\hline \multirow[b]{2}{*}{ ENT } & $\mathrm{ZI}_{1}$ & 3.0 & 0.0 & 5.0 & 16.0 & 0.0 & 3.0 & 0.0 & 0.0 & 8.0 & 0.0 & 2.0 & 4.0 & 10.0 & 0.0 & 18.0 & 7.0 & 3.0 & 26.0 \\
\hline & $\mathrm{ZI}_{2}$ & 0.0 & 0.0 & 16.0 & 15.0 & 0.0 & 2.0 & 5.0 & 15.0 & 20.0 & 0.0 & 4.0 & 4.0 & 4.0 & 0.0 & 21.0 & 3.0 & 2.0 & 25.0 \\
\hline \multirow[b]{2}{*}{ EC } & $\mathrm{ZI}_{1}$ & 10.0 & 0.0 & 15.0 & 10.0 & 6.0 & 0.0 & 0.0 & 11.0 & 10.0 & 0.0 & 1.0 & 5.0 & 8.0 & 0.0 & 10.0 & 0.0 & 15.0 & 16.0 \\
\hline & $\mathrm{ZI}_{2}$ & 5.0 & 0.0 & 16.0 & 18.0 & 3.0 & 0.0 & 0.0 & 17.0 & 14.0 & 0.0 & 3.0 & 2.0 & 7.0 & 0.0 & 18.0 & 0.0 & 16.0 & 19.0 \\
\hline \multirow{4}{*}{ KP } & $\mathrm{ZI}_{1}$ & 3.0 & 2.0 & 0.0 & 10.0 & 0.0 & 0.0 & 9.0 & 12.0 & 13.0 & 0.0 & 3.0 & 2.0 & 12.0 & 0.0 & 19.0 & 0.0 & 10.0 & 30.0 \\
\hline & $\mathrm{ZI}_{2}$ & 1.0 & 3.0 & 0.0 & 7.0 & 0.0 & 0.0 & 11.0 & 1.0 & 15.0 & 0.0 & 0.0 & 2.0 & 14.0 & 0.0 & 20.0 & 0.0 & 12.0 & 28.0 \\
\hline & $\begin{array}{l}\text { Mean } \\
\text { ZI }\end{array}$ & 3.3 & 1.6 & 6.9 & 13.9 & 2.6 & 0.6 & 3.8 & 10.5 & 14.5 & 0.0 & 1.9 & 2.6 & 11.4 & 0.0 & 18.3 & 1.9 & 8.5 & 25.6 \\
\hline & & \multicolumn{10}{|c|}{$6.41 \mathrm{~mm}$} & \multicolumn{8}{|c|}{$10.02 \mathrm{~mm}$} \\
\hline
\end{tabular}


Tables 3 and 4 show the invitro antibiotic sensitivity responses (in terms of zones of inhibition) of four selected pathogens to standard antibiotic discs (Abtek Biological Ltd, Liverpool) and locally manufactured antibiotic discs (Maxicare Medical Laboratory, Nigeria) as carried out on sterile Nutrient and Mueller Hinton agar. Selected antibiotics used for mean inhibition zone comparison were two fluoroquinolones (ie ciprofloxacin and ofloxacin), one aminoglycoside (gentamycin) and a synthetic penicillin - amoxicillin/clavulanic acid. The pathogens used were Pseudomonas aeruginosa (PA), Enterococcus spp (ENTR), Escherichia coli (EC) and Klebsiella pneumoniae (KP).

Table 3 Comparing means of zones of inhibition of standard and local discs on Nutrient agar

\begin{tabular}{|c|c|c|c|c|c|}
\hline \multicolumn{6}{|c|}{ Antibiotics common to both standard and local discs } \\
\hline Selected pathogens & $\begin{array}{c}\text { DISC } \\
\text { CODES }\end{array}$ & $\begin{array}{l}\text { CPX } \\
\text { Mm }\end{array}$ & $\begin{array}{c}\text { AU } \\
\mathbf{m m}\end{array}$ & $\begin{array}{l}\text { OFX } \\
\text { mm }\end{array}$ & $\begin{array}{c}\text { GN } \\
\text { mm }\end{array}$ \\
\hline \multirow[b]{2}{*}{ PA } & STD & 29.0 & 1.5 & 19.0 & 10.0 \\
\hline & LD & 15.0 & 0.0 & 17.0 & 0.0 \\
\hline \multirow[b]{2}{*}{ ENTR } & STD & 24.5 & 4.7 & 25.0 & 15.5 \\
\hline & LD & 21.0 & 1.5 & 18.0 & 12.5 \\
\hline \multirow[b]{2}{*}{ EC } & STD & 15.5 & 0.0 & 15.5 & 5.0 \\
\hline & LD & 5.0 & 0.0 & 14.5 & 0.0 \\
\hline \multirow[b]{2}{*}{$\mathrm{KP}$} & STD & 23.0 & 0.0 & 17.0 & 2.0 \\
\hline & LD & 4.0 & 0.0 & 5.5 & 1.5 \\
\hline \multirow[b]{2}{*}{ Mean \pm SE } & STD & $23.0 \pm 8.9$ & $4.93 \pm 11.25$ & $19.13 \pm 6.64$ & $6.88 \pm 9.70$ \\
\hline & LD & $11.25 \pm 13.01$ & $0.38 \pm 1.19$ & $13.75 \pm 9.06$ & $3.5 \pm 9.61$ \\
\hline $\mathrm{T}_{\text {calculated }}$ & & 1.1081 & 1. 277 & 2.215 & 2.299 \\
\hline \multirow[b]{2}{*}{$\mathrm{P}$-value/T calculated } & $95 \% \mathrm{CI}$ & $P>0.05$ & $P>0.05$ & $P>0.05$ & $\mathrm{P}>0.05$ \\
\hline & $99 \% \mathrm{CI}$ & $\mathrm{P}>0.01$ & $\mathrm{P}>0.01$ & $\mathrm{P}>0.01$ & $\mathrm{P}>0.01$ \\
\hline
\end{tabular}

The mean \pm standard error inhibition zone around the standard ciprofloxacin disc and local ciprofloxacin disc were 23.0 $\pm 8.9 \mathrm{~mm}$ and $11.25 \pm 13.01 \mathrm{~mm}$ respectively s $\mu$ ggesting $14.07 \leq \mu \geq 31.93 \mathrm{~mm}$ and $0.0 \leq \mu \geq 24.26 \mathrm{~mm}$ for standard discs and local discs respectively. A paired T-test at $95 \%$ and $99 \%$ confidence intervals (comparing zones of inhibition of standard discs with those of the local disc) showed that there was no significant difference in their mean \pm standard error zones of inhibition $(\mathrm{P}>0.05, \mathrm{P}>0.01)$ Table 3 .

Mean \pm standard error inhibition zones around standard amoxicillin/clavulanic acid (augmentin) disc and around locally manufactured a $16.18 \mathrm{~mm}$ and $0.0 \leq \mu \geq 1.19 \mathrm{~mm}$ zones of inhibition for the standard and local discs used respectively. At $95 \%$ confidence interval, standard disc inhibition zones were significantly lower than the local disc $(\mathrm{P}<0.05)$. They were however not significantly different at 99\% confidence interval $(\mathrm{P}>0.01)$ Table 3.

Mean \pm standard error inhibition zones of inhibition of selected pathogens by standard and local ofloxacin discs were $19.13 \pm 6.64 \mathrm{~mm}$ and $13.75 \pm 9.06 \mathrm{~mm}$ respectively suggesting $12.49 \leq \mu \geq 25.77 \mathrm{~mm}$ and $4.69 \leq \mu \geq 22.81 \mathrm{~mm}$ zones of inhibition for the standard and local discs used respectively. Paired T-test of inhibition zones of standard and local discs on sterile Nutrient agar showed that at both $95 \%$ and $99 \%$ confidence intervals, inhibition zones of standard and local discs were not significantly different $(\mathrm{P}>0.05, \mathrm{P}>0.01)$ Table 3.

Standard and local gentamycin discs zones of inhibition of selected pathogens were $6.88 \pm 9.70 \mathrm{~mm}$ and $3.5 \pm 9.61 \mathrm{~mm}$ respectively indicating $0.0 \leq \mu \geq 16.58 \mathrm{~mm}$ and $0.0 \leq \mu \geq 13.11 \mathrm{~mm}$ zones of inhibition for the standard and local discs used respectively. A paired T-test of inhibition zones of standard and local discs on sterile Nutrient agar showed that at both $95 \%$ and $99 \%$ confidence intervals, inhibition zones of standard and local discs were not significantly different (P $>0.05, \mathrm{P}>0.01$ ) Table 3 . 
Table 4 Comparing means of zones of inhibition of standard and local discs on Mueller Hinton agar

\begin{tabular}{|c|c|c|c|c|c|}
\hline \multicolumn{6}{|c|}{ Antibiotics common to both standard and local discs } \\
\hline Selected pathogens & $\begin{array}{c}\text { DISC } \\
\text { CODES }\end{array}$ & $\begin{array}{l}\text { CPX } \\
\text { Mm }\end{array}$ & $\begin{array}{l}\mathrm{AU} \\
\mathrm{mm}\end{array}$ & $\begin{array}{l}\text { OFX } \\
\text { mm }\end{array}$ & $\begin{array}{l}\text { GN } \\
\text { mm }\end{array}$ \\
\hline \multirow{2}{*}{ PA } & STD & 30.5 & 2.5 & 20.0 & 18.0 \\
\hline & $\mathrm{LD}$ & 17.5 & 1.0 & 18.0 & 14.0 \\
\hline \multirow[b]{2}{*}{ ENTR } & STD & 25.5 & 5.0 & 19.5 & 7.0 \\
\hline & LD & 5.5. & 2.5 & 14.0 & 2.5 \\
\hline \multirow[b]{2}{*}{$\mathrm{EC}$} & STD & 17.5 & 0.0 & 14.0 & 7.5 \\
\hline & LD & 14.0 & 0.0 & 12.0 & 0.0 \\
\hline \multirow{2}{*}{ KP } & STD & 29.0 & 0.0 & 19.5 & 13.0 \\
\hline & $\mathrm{LD}$ & 8.5 & 0.0 & 14.0 & 10.5 \\
\hline \multirow[b]{2}{*}{ Mean \pm SE } & STD & $25.6 \pm 9.24$ & $1.88 \pm 3.81$ & $18.25 \pm 4.52$ & $11.38 \pm 8.25$ \\
\hline & LD & $11.38 \pm 8.58$ & $0.88 \pm 1.88$ & $14.5 \pm 4.0$ & $6.75 \pm 10.48$ \\
\hline $\mathrm{T}_{\text {calculated }}$ & & 3.589 & 1.633 & 3.711 & 4.411 \\
\hline \multirow{2}{*}{$\mathrm{P}$-value/ $\mathrm{T}_{\text {calculated }}$} & $95 \% \mathrm{CI}$ & $\mathrm{P}<0.05$ & $P>0.05$ & $\mathrm{P}<0.05$ & $\mathrm{P}<0.05$ \\
\hline & $99 \% \mathrm{CI}$ & $P>0.01$ & $\mathrm{P}>0.01$ & $\mathrm{P}>0.01$ & $\mathrm{P}>0.01$ \\
\hline
\end{tabular}

Table 5, the mean \pm standard error zones of inhibition on Mueller Hinton and Nutrient agar of standard and local ciprofloxacin, augmentin, ofloxacin and gentamycin discs are compared. Grand mean \pm standard error zones of inhibition for standard ciprofloxacin, augmentin, ofloxacin and gentamycin discs on Mueller Hinton agar and on Nutrient agar were $14.28 \pm 6.46 \mathrm{~mm}$ and $13.49 \pm 9.13 \mathrm{~mm}$ respectively. On the other hand, Grand mean \pm standard error zones of inhibition for locally manufactured ciprofloxacin, augmentin, ofloxacin and gentamycin discs on Mueller Hinton agar and on Nutrient agar were $8.38 \pm 6.24 \mathrm{~mm}$ and $7.22 \pm 8.22 \mathrm{~mm}$ respectively.

Table 5 Standard and local disc mean zones of inhibition on MHA and NA compared

\begin{tabular}{|c|c|c|c|c|}
\hline \multirow{2}{*}{$\begin{array}{c}\text { Antibiotic } \\
\text { Discs code }\end{array}$} & \multicolumn{2}{|c|}{ Standard discs } & \multicolumn{2}{c|}{ Local discs } \\
\hline & MHA & NA & MHA & NA \\
& Mm & Mm & mm & $11.25 \pm 13.01$ \\
\hline CPX & $25.6 \pm 9.24$ & $23.0 \pm 8.93$ & $11.38 \pm 8.58$ & $0.38 \pm 1.19$ \\
\hline AU & $1.88 \pm 3.81$ & $4.93 \pm 11.25$ & $0.88 \pm 1.88$ & $13.75 \pm 9.06$ \\
\hline OFX & $18.25 \pm 4.52$ & $19.13 \pm 6.64$ & $14.5 \pm 4.0$ & $3.5 \pm 9.61$ \\
\hline GN & $11.38 \pm 8.25$ & $6.88 \pm 9.70$ & $6.75 \pm 10.48$ & $7.22 \pm 8.22$ \\
\hline
\end{tabular}

Table 6 shows a comparison of standard discs inhibition zones on Mueller Hinton agar and Nutrient agar. The Null hypothesis tested stated that "antibiotic sensitivity testing done using standard discs produced inhibition zones NOT significantly different from each other in terms of medium used" Paired T test statistical analysis showed that calculated $\mathrm{T}$ value was lower than $\mathrm{P}$ value (book value) and hence, the null hypothesis is accepted that there is no significant difference in the zones of inhibition in terms of medium inoculated. 
Table 6 Standard discs zones of inhibition on MHA and NA compared

\begin{tabular}{|c|c|c|}
\hline \multirow{2}{*}{ Antibiotic disc codes } & MHA Standard discs \\
\hline CPX & $25.6 \pm 9.24$ & $23.0 \pm 8.93$ \\
\hline AU & $1.88 \pm 3.81$ & $4.93 \pm 11.25$ \\
\hline OFX & $18.25 \pm 4.52$ & $19.13 \pm 6.64$ \\
\hline GN & $11.38 \pm 8.25$ & $6.88 \pm 9.70$ \\
\hline Grand Mean \pm SE & $\mathbf{1 4 . 2 8} \pm \mathbf{6 . 4 6}$ & $\mathbf{1 3 . 4 9} \pm \mathbf{9 . 1 3}$ \\
\hline \multicolumn{2}{|c|}{ Tcalculated $=0.5881 \mathrm{P}-$ value $=3.182$ Paired T test: $\mathrm{P}>0.05$} \\
\hline
\end{tabular}

Accept null hypothesis ie, standard discs zones of inhibition on MHA and NA media are not significantly different. In Table 7, a comparison of locally manufactured discs inhibition zones on Mueller Hinton agar and Nutrient agar is shown. The Null hypothesis tested stated that "antibiotic sensitivity testing done using locally manufactured discs produced inhibition zones NOT significantly different from each other in terms of medium used" Paired T test statistical analysis showed that calculated T value was lower than $\mathrm{P}$ value (book value) and hence, the null hypothesis is accepted that there is no significant difference in the zones of inhibition in terms of medium inoculated.

Table 7 Locally manufactured discs zones of inhibition on MHA and NA compared

\begin{tabular}{|c|c|c|}
\hline \multirow{2}{*}{ Antibiotic disc codes } & \multicolumn{2}{|c|}{ Local discs } \\
\hline CPX & $11.38 \pm 8.58$ & $11.25 \pm 13.01$ \\
\hline AU & $0.88 \pm 1.88$ & $0.38 \pm 1.19$ \\
\hline OFX & $14.5 \pm 4.0$ & $13.75 \pm 9.06$ \\
\hline GN & $6.75 \pm 10.48$ & $3.5 \pm 9.61$ \\
\hline Grand Mean \pm SE & $\mathbf{8 . 3 8} \pm \mathbf{6 . 2 4}$ & $\mathbf{7 . 2 2} \pm \mathbf{8 . 2 2}$ \\
\hline
\end{tabular}

Accept null hypothesis ie, local discs zones of inhibition on MHA and NA media are not significantly different.

Table 8 shows a comparison of standard and locally manufactured discs inhibition zones on Mueller Hinton agar. The Null hypothesis tested stated that "standard and locally manufactured discs produced inhibition zones on Mueller Hinton agar that were NOT significantly different from each other" Paired T test statistical analysis showed that calculated $\mathrm{T}$ value was lower than $\mathrm{P}$ value (book value) and hence, the null hypothesis is accepted that there is no significant difference in the zones of inhibition as obtained on MHA.

Table 8 Standard and local discs mean zones of inhibition on Mueller Hinton agar Compared.

\begin{tabular}{|c|c|c|}
\hline Antibiotic disc codes & $\begin{array}{c}\text { Standard } \\
\text { MHA }\end{array}$ & $\begin{array}{c}\text { Local discs } \\
\text { MHA }\end{array}$ \\
\hline CPX & $25.6 \pm 9.24$ & $11.38 \pm 8.58$ \\
\hline $\mathrm{AU}$ & $1.88 \pm 3.81$ & $0.88 \pm 1.88$ \\
\hline OFX & $18.25 \pm 4.52$ & $14.5 \pm 4.0$ \\
\hline GN & $11.38 \pm 8.25$ & $6.75 \pm 10.48$ \\
\hline Grand Mean \pm SE & $14.28 \pm 6.46$ & $8.38 \pm 6.24$ \\
\hline
\end{tabular}

Accept null hypothesis ie, local discs zones of inhibition on MHA and NA media are not significantly different. 
Finally, Table 9 shows a comparison of standard and locally manufactured discs inhibition zones on Nutrient agar. The Null hypothesis tested stated that "standard and locally manufactured discs produced inhibition zones on Nutrient agar that were NOT significantly different from each other" Paired T test statistical analysis showed that calculated T value was lower than P value (book value) and hence, the null hypothesis is accepted that there is no significant difference in the zones of inhibition as obtained on MHA.

Table 9 Standard and local discs mean zones of inhibition on Nutrient agar compared

\begin{tabular}{|c|c|c|}
\hline $\begin{array}{c}\text { ANTIBIOTIC DISC } \\
\text { CODES }\end{array}$ & STANDARD & LOCAL DISCS \\
NA \\
\hline CPX & $23.0 \pm 8.93$ & $11.25 \pm 13.01$ \\
\hline AU & $4.93 \pm 11.25$ & $0.38 \pm 1.19$ \\
\hline OFX & $19.13 \pm 6.64$ & $13.75 \pm 9.06$ \\
\hline GN & $6.88 \pm 9.70$ & $3.5 \pm 9.61$ \\
\hline Grand Mean \pm SE & $\mathbf{1 3 . 4 9} \pm \mathbf{9 . 1 3}$ & $\mathbf{7 . 2 2} \pm \mathbf{8 . 2 2}$ \\
\hline \multicolumn{2}{|c|}{$\mathrm{T}_{\text {calculated }}=2.043 \mathrm{P}-$ value $=3.182$ Paired T test: $\mathrm{P}>0.05$} \\
\hline
\end{tabular}

Accept null hypothesis ie, local discs zones of inhibition on MHA and NA media are not significantly different.

Inhibition zones around selected pathogens by standard and local antibiotic discs on sterile Mueller Hinton agar medium were also recorded. The mean \pm standard error zone of inhibition of standard and local ciprofloxacin discs as recorded on Mueller Hinton agar were $25.6 \pm 9.24 \mathrm{~mm}$ and $11.38 \pm 8.58 \mathrm{~mm}$ respectively s $\mu$ ggesting $16.36 \leq \mu \geq 34.84 \mathrm{~mm}$ and $2.80 \leq \mu \geq 19.96 \mathrm{~mm}$ zones of inhibition for the standard and local discs used respectively. A paired T-test of inhibition zones of ciprofloxacin standard and local discs on Mueller Hinton agar showed that at $95 \%$ confidence interval, inhibition zones of standard and local discs were significantly different $(\mathrm{P}<0.05)$ but were not significantly different at 99\% confidence interval $(\mathrm{P}>0.01)$ Table 4.

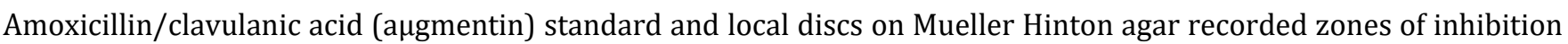
of $1.88 \pm 3.81 \mathrm{~mm}$ and $0.88 \pm 1.88 \mathrm{~mm}$ respectively suggesting $0.0 \leq \mu \geq 5.69 \mathrm{~mm}$ and $0.0 \leq \mu \geq 2.76 \mathrm{~mm}$ zones of inhibition for the standard and local discs used respectively. A paired T-test of standard and local discs inhibition zones showed that there was no significant difference between them at $95 \%$ and $99 \%$ confidence intervals $(\mathrm{P}<0.05, \mathrm{P}>0.01)$ Table 4.

The mean \pm standard error zones of inhibition of ofloxacin standard and local discs was $18.25 \pm 4.52 \mathrm{~mm}$ and $14.5 \pm$ $4.0 \mathrm{~mm}$ respectively indicating $13.73 \leq \mu \geq 22.77 \mathrm{~mm}$ and $10.5 \leq \mu \geq 18.5 \mathrm{~mm}$ zones of inhibition for the standard and local discs used respectively. Ofloxacin standard and local discs paired T-test zones of inhibition suggested that there was a significant difference at $95 \%$ confidence interval $(\mathrm{P}<0.05)$ while there was no significant difference at $99 \%$ confidence interval $(\mathrm{P}>0.01)$ Table 4 .

Gentamycin standard and local discs on sterile Mueller Hinton agar recorded zones of inhibition of $11.38 \pm 8.25 \mathrm{~mm}$ and $6.75 \pm 10.48 \mathrm{~mm}$ respectively suggesting a range of $3.13 \leq \mu \geq 19.63 \mathrm{~mm}$ and $0.0 \leq \mu \geq 17.23 \mathrm{~mm}$ for standard and local discs used respectively. A paired T-test of gentamycin standard and local discs zones of inhibition showed that there was a significant difference at $95 \%$ confidence interval $(\mathrm{P}<0.05)$ whereas, at $99 \%$ confidence interval, there was no significant difference ( $\mathrm{P}>0.01)$ Table 4 .

\section{Discussion}

Nutrient agar is an all-purpose medium (a general purpose) nutrient medium used for the cultivation of microorganisms and for supporting growth of a wide range of non-fastidious organisms. It is a popular medium because it can grow a variety of different types of bacteria and fungi.

Mueller Hinton agar (MHA) on the other hand is a loose agar that allows for better diffusion of antibiotics than most other media and a better diffusion leads to a truer zone of inhibition [8]. Studies have established that commercially manufactured MHA is suitable for use in routine susceptibility testing [18]. In addition, the Hardy Diagnostics Mueller Hinton agar meets the standard performance established by the Clinical and Laboratory Standards Institute [7, 11]. 
Mueller Hinton medium contains beef infusion, casamino acids and starch. Starch acts as a colloid that protects against toxic materials in the medium. Beef infusion and casamino acids provide energy and nutrients. Agar is added when a solidifying agent is needed. The levels of tetracycline and sulfonamide inhibitors, thymidine, thymine, magnesium and calcium ions are controlled so as not to interfere with susceptibility testing and to yield good growth [19]. Mueller Hinton Broth (MHB) is the same formulation but without the added agar. It is used for the cultivation of microorganisms and for making dilutions of organisms used in the Bauer-Kirby diffusion procedure.

Common sources of error encountered in clinical Microbiology laboratory which may compromise the reliability and accuracy of AST results include improper disc storage, inoculum not properly adjusted (being too light or too heavy), incubation temperature deviating from $35-37^{\circ} \mathrm{C}$, use of an increased $\mathrm{CO}_{2}$ atmosphere, reading plates before or after $16-$ $18 \mathrm{hrs}$ incubation, transcribing errors, reader error when measuring zone diameters, deterioration of the McFarland turbidity standard and contamination or mutation in the control strains [20].

The selection of an appropriate antimicrobial is a challenging task for clinicians [1]. The Bauer-Kirby diffusion method is one of the most widely practiced antimicrobial susceptibility tests (AST) and it is affected by many factors among which, are type of media used [1]. When the Bauer-Kirby test is performed using a standard procedure, it gives reliable results and can predict clinical efficacy of the antibiotics tested [4,5]. The validity of AST depends on rigid standardization of every feature of the test particularly the composition of the medium used [6]. Due to ease of obtaining quality media and financial issues (cost of media), MHA is not a feasible option in many developing countries and instead, NA is used for AST [7].

This study did not carry out internal Quality Control (QC) of AST using standard discs and reference organisms (as control organisms) in line with CLSI guidelines. This work did not also address CLSI guidelines in terms of assessing very major error (false sensitive) or major error (false resistant) patterns. According to CLSI, in a standard AST, very major errors patterns should not exceed $1.5 \%$ while major errors should not exceed $3.0 \%[10,9,11]$.

Rather, in this study, the focus was on using Paired T-test at 95\% and 99\% confidence intervals to know if there is a significant (or otherwise) performance of selected standard antibiotic discs over selected locally manufactured discs on NA on the one hand, and standard discs over local discs on MHA on the other hand. The study also sought to know if standard discs will give better inhibition zones on MHA over NA and lastly, if local discs performance will be better on MHA or NA and vice versa.

Table 1 shows that locally manufactured ofloxacin and ciprofloxacin (both fluoroquinolones) recorded the highest mean ZI of $12.25 \mathrm{~mm}$ and $9.25 \mathrm{~mm}$ respectively while streptomycin recorded zero ZI and the least ZI was recorded by

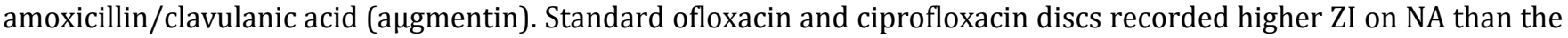
locally made fluoroquinolones on the same NA medium. This was further buttressed by an overall mean ZI of 5.31mm recorded by local discs over $8.13 \mathrm{~mm}$ overall mean ZI recorded by the standard discs. This finding implies that if at all, Nutrient agar happens to be the only available medium for AST at any time, standard discs will produce better and more reliable AST result patterns.

The results in Table 2 clearly show an enhanced AST results on MHA. As in Table 1, the highest mean ZIs of 14.5mm and $13.9 \mathrm{~mm}$ were recorded by local ofloxacin and ciprofloxacin discs respectively on MHA over $18.3 \mathrm{~mm}$ and $25.6 \mathrm{~mm}$ mean ZIs recorded by the same fluoroquinolones on the same medium. These patterns were further elucidated by overall mean ZI of $6.41 \mathrm{~mm}$ recorded by local discs over $10.02 \mathrm{~mm}$ overall mean ZI recorded by the standard discs on MHA medium.

Again, the antibiograms recorded on MHA suggest that better and more reliable ZIs will be recorded by standard discs over local discs and also, better and more reliable ZIs will be achieved when MHA is used as the medium. This is because, ordinarily, standard discs produced overall mean ZI of $10.02 \mathrm{~mm}$ on MHA over overall mean ZI of 8.13mm on NA. The local discs also yielded higher mean ZI on MHA compared to mean ZI recorded on NA. These results however, may not be statistically different from each other. According to Donkor et al. [12], overall discrepancy in susceptibility results between NA and MHA discouraged the use of NA in the Bauer-Kirby agar diffusion method of AST as practiced by some clinical laboratories.

In this study, the performance of MAXI discs (a locally manufactured brand of antibiotic disc) was compared with that of ABTEK disc (a standardized foreign disc) on two media - Nutrient agar and Mueller Hinton agar. The MAXI disc brand incorporated a total of ten discs while the standard disc brand incorporated eight discs. Besides, the local brand used in this study contained some codes different from those adopted by the standard discs. For instance, whereas the local brand used CPX and OFX for ciprofloxacin and ofloxacin, the standard disc brand adopted CPR and OFL for the same 
antibiotics respectively. While the use of different codes may not be much of an issue, the number of discs on a multidisc panel for a $90 \mathrm{~mm}$ Petri dish should conform to International Standards in line with the recommendation of WHO of six or not more than eight discs per plate as against ten discs incorporated into the local discs used in this study. The excess of two discs may have accounted for un-interpretable zones of inhibition (i.e large zones of inhibition that merged together or completely wiped out bacterial growth at the time of reading the plates) which were encountered in the course of reading the antibiograms. Some authors who did a related study also reported this experience [21].

Another discrepancy was the issue of concentrations of individual antibiotics. Again, for example, whereas ciprofloxacin, amoxicillin/clavulanic acid, ofloxacin and gentamycin local brand discs contained 30 $\mu \mathrm{g}, 10 \mu \mathrm{g}, 10 \mu \mathrm{g}$ and $30 \mu \mathrm{g}$ concentrations of the drugs, the same antibiotics of the standard discs brand contained $5 \mu \mathrm{g}, 30 \mu \mathrm{g}$, $5 \mu \mathrm{g}$ and $10 \mu \mathrm{g}$ concentrations respectively of the antibiotics. The use of different codes and in some cases, different concentrations for the same antibiotics (as in brands being used in this study) reflects a state of much confusion which makes it difficult to make a direct and comprehensive comparison of the performance of these two brands. Ekundayo and Omodamiro [21] also reported similar challenges in their related study. These discrepancies may be a reflection of lack of clear policy guidelines on antibiotic usage or failure of manufacturers to comply with such guidelines.

The clinical implication of higher concentrations of antibiotics above the required or recommended concentrations in sensitivity discs is that misleading results might be sent to clinicians (physicians) based on wrong antibiograms or results being produced by the discs. A bacterial strain may be recorded as sensitive instead of resistant strain and vice versa. Such false results could be used as the basis for antibiotic prescription by the physician. This will be of no benefit to the patient with the attendant adverse side effects and possible long hospital stay as well as wastage of resources used in buying such drugs. It can further contribute to the problem of misuse of antibiotics (Ibeawuchi and Mbata, [22] which favors the emergence of $\mathrm{dr} \mu \mathrm{g}$ resistant strains of bacteria [23].

Notwithstanding the highlighted challenges, the performance of the two brands of antibiotic discs on NA and MHA was compared by using four antibiotics common to both and these were ciprofloxacin, augmentin, ofloxacin and gentamycin as presented in Tables 6-9. The mean \pm standard error zones of inhibition of Pseudomonas aeruginosa, Enterococcus spp, Escherichia coli and Klebsiella pneumoniae on Nutrient agar to standard and local ciprofloxacin antibiotic were $23.0 \pm$ $8.9 \mathrm{~mm}$ and $11.3 \pm 13.1 \mathrm{~mm}$ respectively. Standard a gmentin, ofloxacin and gentamycin antibiotic discs recorded higher zones of inhibition of $4.9 \pm 11.3 \mathrm{~mm}, 19.1 \pm 6.6$ and $6.9 \pm 9.7 \mathrm{~mm}$ respectively over the local discs containing the same antibiotics of $0.38 \pm 1.2 \mathrm{~mm}, 13.8 \pm 9.1 \mathrm{~mm}$ and $3.5 \pm 9.6 \mathrm{~mm}$ respectively. Hence, on Nutrient agar, Abtek standard ciprofloxacin, ofloxacin and gentamycin antibiotic discs produced higher diameter zones of inhibition than the corresponding local antibiotic discs which were not significantly different statistically $(\mathrm{P}>0.05)$.

The differences however in inhibition diameter zones were not statistically significant. This might be attributable to the fact that three out of the four selected local disc antibiotics contained higher concentrations of the antibiotics than those of the Abtek standard discs.

The outcome of the performance comparison was clearly diffserent on Mueller Hinton agar (Table 4). Abtek standard ciprofloxacin, ofloxacin and gentamycin produced mean \pm standard error zones of inhibition which were significantly higher than those produced by MAXI disc brand $(\mathrm{P}<0.05)$. This finding suggests or emphasizes that standard discs will perform better than local disc on MHA. The differences in antibiotic concentrations as stated above may account for why at 99\% confidence, the differences in standard and local disc zones of inhibition were not significant (P > 0.01).

The performance of Abtek standard discs on MHA and NA ordinarily showed that standard discs produced higher zones of inhibition on MHA than NA but the difference was not statistically different $(P>0.05)$ Table 6 . Although the implication of this is that AST done on MHA with standard discs will not yield much of a difference than AST done on NA using the same standard discs, this inference cannot be taken hookline and sinker because other factors (apart from type of media) such as inoculum size, incubation conditions, irregular power supply etc may have affected the results [4].

Similarly, the MAXI discs (local discs) recorded slightly better performance on MHA than on NA although the difference in performance (zones of inhibition) was not significantly different statistically (P > 0.05). Table 7. Despite the slight difference, it points to the fact that MHA will yield a better AST result.

The outcome or performance of standard and local discs on MHA on the one hand and standard/local discs performance on NA on the other hand, showed a better performance of standard discs on MHA compared to local discs and in the absence or non-availability of MHA, standard discs will produce better zones of inhibition on NA compared to local discs. These results were however not significantly different from each other statistically $(\mathrm{P}>0.05)$. Tables 8 and 9 . 


\section{Conclusion}

Although the findings of this study cannot be effectively used to generalize on the performance of MHA over NA or on the performance of standard discs over locally manufactured discs owing to the discrepancies already highlighted, they notwithstanding, establish a basis for further and more elaborate research in the same direction. Besides, Abtek standard ciprofloxacin, ofloxacin and gentamycin discs (or perhaps, all standard discs) produced mean \pm standard error zones of inhibition which were significantly higher (on MHA) and not significantly higher (on NA) than those produced by the corresponding local (MAXI) disc brand. Abtek standard discs produced better and more reliable AST results on MHA than local discs and if at all Nutrient agar happens to be the only available agar for AST at any time, standard discs will produce better and more reliable antibiograms. Also, owing to disparity in use of antibiotic codes and differences in minimum inhibitory concentrations (MIC) of antibiotics used, there is need for manufacturers of local discs to strictly adopt and apply guidelines and procedures for standardization of their products. This will help to make them more competitively acceptable moreso as the standard discs are almost out of reach due to their huge cost.

\section{Recommendation for further studies}

In any future study, reference strains or organisms should be run as controls side by side the test organisms. This will make for effective comparison in line with CLSI guidelines in order to detect false sensitive and false resistant antibiotic occurrences.

Internal Quality Control of AST should be run using standard discs and reference organisms in line with CLSI guidelines.

Sample size in terms of clinical isolates should be increased.

More local discs (properly standardized local discs) should be used.

\section{Compliance with ethical standards}

\section{Acknowledgments}

We hereby appreciate laboratory staff of Microbiology Department of Western Delta University, Oghara, Nigeria. We also thankfully acknowledge Chrida Medical Laboratory Services, Benin City, Nigeria for helping with the diagnostic and antibiotic analyses.

\section{Disclosure of conflict of interest}

We hereby state that there is no conflict of interest of any kind.

\section{References}

[1] Nasser MS, Hazzah WA.and Bakr MK. Validation of AST results on NA medium as a substitute for MHA by some Microbiology laboratories in Alexandria, Egypt. Journal Egypt Public Health Association, 2019; 94 (1):4-10.

[2] FDA. 2017. US battle of the bugs: fighting antibiotic resistance. [Internet]Updated: Available from:http://www.fda.gov/drugs/resourcesforyou/consumers/ucm143568.htm

[3] Shigemura k, Tanaka k, Adachi M, Yamshita M, Arakawa S. and FujisawaM. Chronological change of antibiotic use and antibiotic resistance in Escherichia coli causing urinary tract infections. Journal Infectious Disease and Chemotherapy, 2011; 17:646-651. Doi :10.1007/s10156-011-0241-

[4] King A. and Brown DFJ. Quality assurance of antimicrobial susceptibility testing by disc diffusion. Journal of Antimicrobial Chemotherapy, 2001; 48:71-76. doi: 10.1093/jac/48.suppl.

[5] Clinical \& Laboratory Standards Institute Performance standards for antimicrobial susceptibility testing; 20th Informational supplement M100-S20. Wayne: CLSI. 2010

[6] Garrod LP. and Waterworth PM. A study of antibiotic sensitivitytesting with proposals for simple uniform methods. Journal of Clinical Patholo Pathology, 1971; 24:779-789. doi: 10.1136/jcp.24.9.779.

[7] Niederstebruch N. and Sixt D. Standard nutrient agar 1 as a substitute for blood-supplemented Müeller-Hinton agar for antibiograms in developing countries. European Journal of Clinical Microbiology and Infectious Disease, 2013; 32:237-241. doi: 10.1007/s10096-012-1735-2. 
[8] ARYAL, S. (2015). Mueller Hinton agar (MHA) - composition, principle, uses and preparation. Microbiology info. [Internet]Availablefrom:http://www.microbiologyinfo.com/mueller-hinton-agar-mha composition principle uses-and-preparation.

[9] Jean SS, Liao CH, Sheng WH, Lee WS. and Hsueh PR.Comparison_of commonly used antimicrobial susceptibility testing' methods for evaluating_susceptibilities of clinical isolates of Enterobacteriaceae and non-fermentative Gram-negative bacilli to cefoperazone-sulbactam. Journal of Microbiology, Immunology and Infection, 2017; 50(4):454-453.

[10] Stuckey S. Automated systems: an overview. In: Schwalbe R, Steele-Moore L, Goodwin AC, editors. Antimicrobial susceptibility testing protocols. Ch 5. Boca Raton: CRC Press Taylor and Francis; 2007; 81-89pp.

[11] Clinical and Laboratory Standards Institute 2007. Methods for dilution and antimicrobial susceptibility tests for bacteria that grow aerobically, approve approved standard M7-A7. Wayne: CLSI.

[12] Donkor ON, Henrickson A and Singh KT. ACE- inhibitory activit activity of probiotic Yoghurt. International Dairy Journal, 2007; 17(11):1321-1331.

[13] Cullimoore DR. 2000. Practical atlas for bacterial identification.Lewis Publishers, Boca Raton. 102p.

[14] Cowan ST. and Steel KJ. Manual for the identification of medical medical Bacteria. $3^{\text {rd }}$ edn. Cambridge University Press. Lonon, New York, Rockville, Melbourne and Sydney. 150p 1993

[15] Bauer AW, Kirby WMM, Sherries JC.and Turck M. Antibiotic susceptibility testing by a standardized single Disk Method. American Journal of Clinical Pathology, 1966; 45:493-496.

[16] Ochei J. and Kolhatka, C. Medical laboratory science theory and practice. New Delhi: Tata Mc Graw hill publishing company limited. 2008; 233-255pp.

[17] Byron F, Brehm S. and Eric AJ. Sensitization of Staphylococcus aureus and Escherichia coli to antibiotics by these sesquiterpenoids. Antimicrob Antimicrobial Agents and Chemotherapys, 2003; 47(10):3357-3360.

[18] Mueller JH.and Hinton J. A protein-free medium for primary isolation of Gonococcus, Meningococcus. Proc. Soc. Exp. Diol and Med, 1941; 48:30-333.

[19] Haltiner RC, Migneault PC and Robertson RG. Incidence of thymidine Dependent enterococci detected on Mueller Hinton agar with thymidine content. Antimicrobial Agents and Chemotherapy, 1980; 18(3):365-368.

[20] Boot R. Frequent major errors in antimicrobial susceptibility testing of bacterial strains distributed under the Deutsches Quality Assurance Program. Laboratory Animal, 2012; 46(3):253-257.

[21] Ekundayo EO. and Omodamiro DO. Evaluation of the quality of locally manufactured antimicrobial susceptibility testing discs used in South Eastern Nigeria. African Journal of Clinical and Experimental Microbiology, 2008; 9(3):122-128.

[22] Ibeawuchi R. and Mbata IJ. Rational and irrational use of antibiotics. Africa Health, 2000; 24(2):16-17.

[23] World Health Organization. Antimicrobial resistance: Fact sheet.No194. 2005 